\title{
A Brief Introduction to DQ Tau
}

\author{
Keivan G. Stassun \\ University of Wisconsin, 475 N. Charter St., Madison, WI 53706, USA
}

\begin{abstract}
We review the salient observational properties of DQ Tau, with an emphasis on the photometric and spectroscopic time-variability of the system. We summarize the evidence for this variability being linked to the orbital dynamics of the underlying close, eccentric, binary.
\end{abstract}

\section{Introduction}

The article by R. Mathieu (this volume) features DQ Tau as an exemplar for the evolution of disks and accretion in close binary systems. This article is intended to provide a brief introductory review of the DQ Tau system-its basic stellar properties, its binary nature, and the peculiar time-variable behavior that makes this system such an important one for understanding the nature of accretion in close binaries. The primary references for the observations summarized here are Mathieu et al. (1997) and Basri et al. (1997).

\section{Observed Properties of DQ Tau}

\subsection{A "Classical" Classical T Tauri Star?}

As pre-main sequence stars go, DQ Tau is at first sight a somewhat unremarkable object. It has long been considered a fairly typical Classical $\mathrm{T}$ Tauri Star (CTTS) as it possesses all of the canonical, defining characteristics of a CTTS: late spectral type $(\sim \mathrm{K} 7)$, photometric variability $(\Delta V \sim 0.5 \mathrm{mag})$, strong $\mathrm{H} \alpha$ emission $(\mathrm{EQW} \sim 200 \AA)$, and large infrared excess $(K-N \approx 4.2)$. These are typically taken as indicators of active accretion from a circumstellar disk. DQ Tau's inferred disk accretion rate is $\sim$ few $\times 10^{-8} \mathrm{M}_{\odot} \mathrm{yr}^{-1}$, a typical value among CTTS.

\subsection{A CTTS SB2 - not so Typical after all!}

What stands DQ Tau apart is its binary nature. DQ Tau is in fact a doublelined spectroscopic binary; only two other double-lined spectroscopic binaries are known among the CTTS (UZ Tau E and AK Sco; see article by E. Jensen, this volume). The orbital solution for DQ Tau is shown in Fig. 1. DQ Tau is a short-period, eccentric binary, with roughly equal-mass components (Table 1).

Given these orbital parameters, the components of the DQ Tau binary have an extremely small spatial separation at periastron: only $\sim 8 R_{\star}$ ! This poses a tantalizing mystery: How are the components of the DQ Tau system able to 


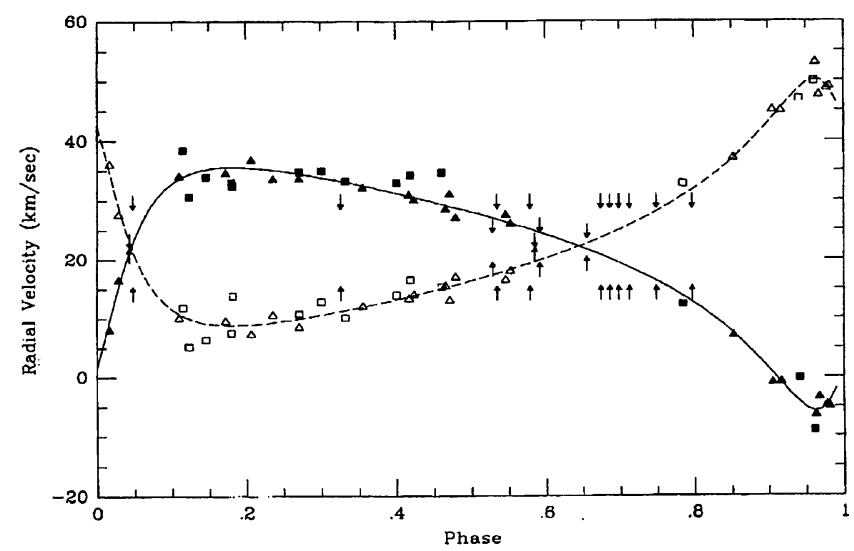

Figure 1. The orbital solution of the DQ Tau SB2 system (Mathieu et al. 1997). Data from MMT, Lick, Keck, McDonald observatories.

\begin{tabular}{lc}
\hline$P_{\text {orb }}$ & 15.8 days \\
$e$ & 0.56 \\
$q$ & $\approx 1.0$ \\
\hline
\end{tabular}

Table 1. Orbital period, eccentricity, and mass ratio of the DQ Tau binary system.

continue accreting? How is circumstellar material replenished in the presence of a close binary companion? What does this tell us about the physics of accretion flows in close binary systems more generally?

\section{A Clue: Periodic "Accretion Events" in DQ Tau}

The solution to the mystery of continued active accretion in such a close binary may lie in the peculiar photometric and spectroscopic variability that has been observed in the DQ Tau system. As we now discuss, the observed photometric variability and the spectroscopic variability apparently possess a common origin that is linked to the orbital dynamics of the binary.

\subsection{Periodic Photometric Brightenings}

DQ Tau owes its status as a CTTS in part to its photometric variability. Though largely stochastic in nature, this photometric variability in fact possesses a statistically significant periodic component (Mathieu et al. 1997). In particular, DQ Tau exhibits brightening events (Fig. 2) which recur quasi-periodically with the same period as the binary orbital period, 15.8 days. These brightening events are brief in duration, during which time the system's integrated light becomes both brighter and bluer. Most importantly, the observed brightenings are synchronized with periastron of the binary (Fig. 3). 


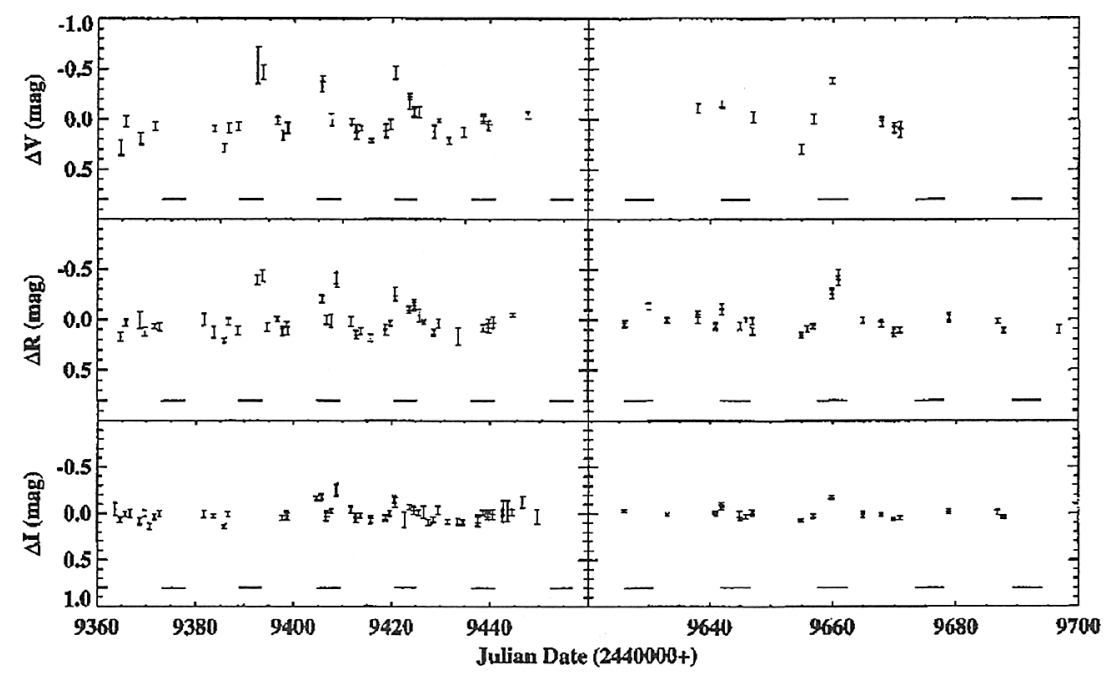

Figure 2. $\quad V R I$ light curves of DQ Tau from Mathieu et al. (1997). Note the quasi-periodically recurring brightening events. Horizontal marks indicate a range of 0.25 phase about periastron; brightening events, when they occur, occur around closest passage of the binary. Brightening events occur at least $65 \%$ of the time, but not always.

\subsection{Periodic Spectroscopic Emission/Veiling}

DQ Tau similarly exhibits periodicity in its spectroscopic variability (Basri et al. 1997). In particular, $\mathrm{H} \alpha$ equivalent width, and spectroscopic veiling, vary during the binary orbit. As with the photometric variability of the system, these spectroscopic signatures of accretion become most pronounced during periastron (Fig. 4).

\section{DQ Tau: Exemplar for Orbitally Driven Accretion in Close Bina- ries}

These photometric and spectroscopic observations provide strong evidence for punctuated episodes of accretion that are causally linked to the orbital dynamics of the DQ Tau binary system. DQ Tau thus provides important observational constraints for models of accretion and disk evolution in close binaries. The implications of DQ Tau for specific models of accretion and disk evolution in close binaries are discussed in more detail by R. Mathieu (this volume).

\section{References}

Basri, G., Johns-Krull, C. M., \& Mathieu, R. D. 1997, AJ, 114, 781

Mathieu, R. D., Stassun, K. G., Basri, G., Jensen, E. L. N., Johns-Krull, C. M., Valenti, J. A., \& Hartmann, L. W. 1997, AJ, 113, 1841 


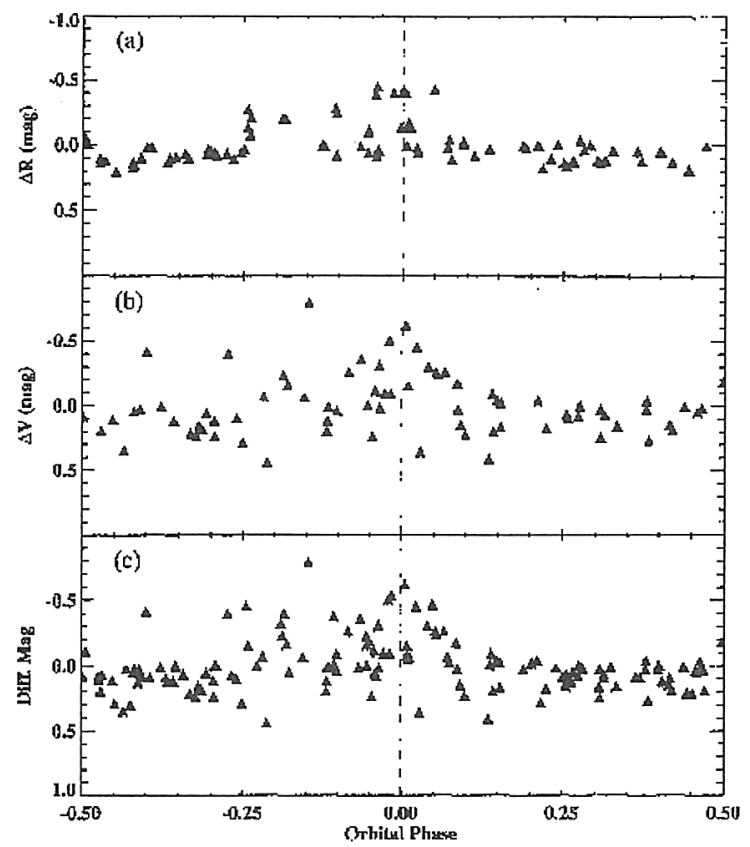

Figure 3. Light curves of DQ Tau phased on the orbital period of 15.8 days. Phase zero indicates periastron. Photometric brightening events occur near periastron, but over a range of about 0.25 phase. The process driving these brightenings is apparently somewhat stochastic.
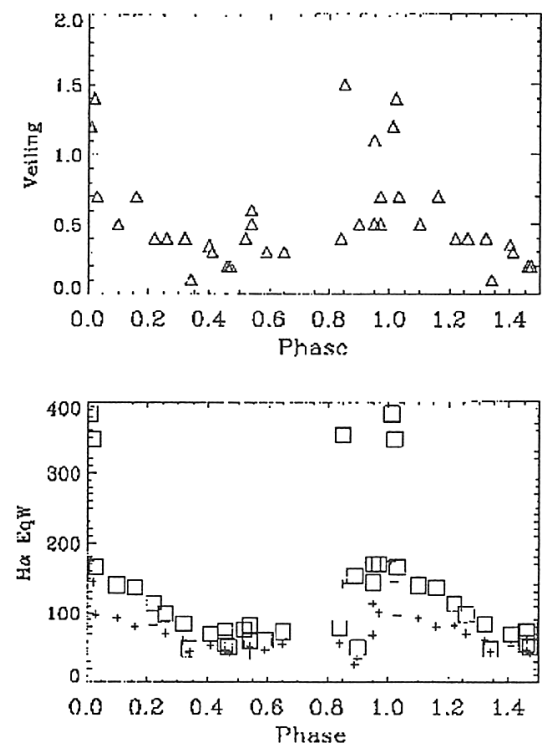

Figure 4. Spectroscopic veiling and $\mathrm{H} \alpha$ equivalent width measurements are shown phased on the binary orbital period of 15.8 days. Phase zero corresponds to periastron. Though characterized by some stochasiticity, both diagnostics are clearly strongest near periastron. 